PROCEEDINGS OF THE

AMERICAN MATHEMATICAL SOCIETY

Volume 129, Number 7 , Pages 1987-1993

S 0002-9939(00)05774-

Article electronically published on December 13, 2000

\title{
LINEAR DISCRETE OPERATORS ON THE DISK ALGEBRA
}

\author{
IVAN V. IVANOV AND BORIS SHEKHTMAN
}

(Communicated by Dale Alspach)

\begin{abstract}
Let $\mathcal{A}$ be the disk algebra. In this paper we address the following question: Under what conditions on the points $z_{k, n} \in \mathbf{D}$ do there exist operators $L_{n}: \mathcal{A} \rightarrow \mathcal{A}$ such that$$
L_{n} f=\sum_{k=1}^{m_{n}} f\left(z_{k, n}\right) l_{k, n}, \quad f, l_{k, n} \in \mathcal{A},
$$

and $L_{n} f \rightarrow f, n \rightarrow \infty$, for every $f \in \mathcal{A}$ ? Here the convergence is understood in the sense of sup norm in $\mathcal{A}$. Our first result shows that if $z_{k, n}$ satisfy Carleson condition, then there exists a function $f \in \mathcal{A}$ such that $L_{n} f \nrightarrow f$, $n \rightarrow \infty$. This is a non-trivial generalization of results of Somorjai (1980) and Partington (1997). It also provides a partial converse to a result of Totik (1984). The second result of this paper shows that if $L_{n}$ are required to be projections, then for any choice of $z_{k, n}$ the operators $L_{n}$ do not converge to the identity operator. This theorem generalizes the famous theorem of Faber and implies that the disk algebra does not have an interpolating basis.
\end{abstract}

\section{IntRoduction}

The problem of recovery of a continuous function from its values at a discrete number of points is well studied in approximation theory. Many forms of linear operators (cf. Bernstein polynomials, spline interpolation) exist that realize such recovery. In contrast very little is known about the recovery of functions in the disk-algebra. In this paper we study the existence of linear operators on the diskalgebra that recover functions from their values at a discrete number of points in the closed unit disk $\mathbf{D}=\{z \in \mathbf{C},|z| \leq 1\}$. The first result in this direction was done by Somorjai in 1980 .

Let $\mathbf{T}=\{z \in \mathbf{C},|z|=1\}$ be the unit circle and $\mathcal{A}$ be the disk-algebra, i.e. the set of all functions $f$ analytic inside and continuous on the boundary of $\mathbf{D}$.

Definition 1. For a given subset $Z \subset \mathbf{D}$ let $R(Z)$ be the collection of all linear continuous operators $L$ on $\mathcal{A}$ that satisfy:

$$
\text { If } f, g \in \mathcal{A} \quad \text { and } \quad f_{\mid Z}=g_{\mid Z} \text {, then } \quad L f=L g .
$$

Theorem 1 ([So] $)$. Let $\left\{Z_{n}\right\}_{n=1}^{\infty}$ be an arbitrary sequence of finite subsets of the unit circle $\mathbf{T}$. Let $L_{n} \in R\left(Z_{n}\right)$. Then there exists a function $f$ in the disk-algebra

Received by the editors November 30, 1998 and, in revised form, October 15, 1999.

2000 Mathematics Subject Classification. Primary 46-XX.

The results in this paper are part of the Ph.D. thesis of the first author.

(C)2000 American Mathematical Society 
$\mathcal{A}$ such that

$$
L_{n} f \nrightarrow f, \quad n \rightarrow \infty .
$$

In 1984 Totik showed that Somorjai's Theorem is not valid when the sets $Z_{n}$ are chosen to be inside the open unit disk $\mathbf{D}^{o}$. More precisely,

Theorem 2 ([] $]$ ). Let $\left\{Z_{n}\right\} \subset \mathbf{D}^{\mathbf{o}}$ satisfy

$$
\sum_{z \in Z_{n}}(1-|z|) \rightarrow \infty, \quad \text { as } n \rightarrow \infty
$$

Then there exist $L_{n} \in R\left(Z_{n}\right)$ such that

$$
L_{n} f \rightarrow f, \quad n \rightarrow \infty \text { for all } f \in \mathcal{A} .
$$

The necessary and sufficient conditions on the sets $Z_{n}$ that guarantee the existence of operators $L_{n} \in R\left(Z_{n}\right)$ satisfying (4) is not known. In this paper we prove

Theorem 3. Let $\left\{Z_{n}\right\}_{n>0}$ satisfy the uniform Carleson condition (cf. Definition 6). Then for every sequence $L_{n} \in R\left(Z_{n}\right)$ there is a function $f \in \mathcal{A}$ such that $L_{n} f \nrightarrow f$ as $n \rightarrow \infty$.

Hence condition (3) is very close to being necessary and sufficient. In section 3 we use this theorem to prove a surprising

Theorem 4. Let $\left\{Z_{n}\right\} \subset \mathbf{D}$ be arbitrary finite sets and let $P_{n} \in R\left(Z_{n}\right)$ be projections on $\mathcal{A}$. Then there is a $f \in \mathcal{A}$ such that $P_{n} f \nrightarrow f$, as $n \rightarrow \infty$.

In particular it implies that $\mathcal{A}$ does not possess an interpolating basis (cf. [Bo]), i.e. a Schauder basis with the property that for every $f \in \mathcal{A}$ the partial sums from its basis representation interpolate $f$ at a certain collection of points in the unit disk $\mathbf{D}$. The proof of Theorem 3 relies heavily on the technique of factorization of operators borrowed from the Banach space theory. The application of that technique to the recovery problem was first noted in [Sh and continued in [Sh]. The proof of Theorem 4 combines the result of Theorem 3 and the analysis of the projections on $\mathcal{A}$ done in $\mathrm{CPS}$. We will use the rest of this section to introduce a few definitions and theorems that are needed in the proofs of Theorems 3 and 4 .

Definition 2. A collection $\left\{z_{i, n}\right\}_{i=1}^{m_{n}}, n=1,2, \ldots$, of points in the unit disk $\mathbf{D}$ is called uniformly Blaschke iff there is a constant $C$ such that $s_{n}=\sum_{i=1}^{m_{n}}\left(1-\left|z_{i, n}\right|\right) \leq$ $C$ for all $n$.

Definition 3. A collection $\left\{z_{i, n}\right\}_{i=1}^{m_{n}}, n=1,2, \ldots$, of points in the unit disk $\mathbf{D}$ is called uniformly non-Blaschke iff $s_{n}=\sum_{i=1}^{m_{n}}\left(1-\left|z_{i, n}\right|\right) \rightarrow \infty$, as $n \rightarrow \infty$.

Definition 4. Let $A: X \rightarrow X$ where $X$ is a Banach space, and define

$$
\gamma_{\infty}(A)=\inf \left\{\|U\|\|V\|: \quad U: X \rightarrow l_{\infty}^{N}, V: l_{\infty}^{N} \rightarrow X, A=U V\right\}
$$

where inf is taken over all positive integers $N$ and all possible factorizations of $A$.

The observation is:

Proposition 1 ([Sh] $)$. Suppose $A_{n}: \mathcal{A} \rightarrow \mathcal{A}$, and $\gamma_{\infty}\left(A_{n}\right)=O(1)$. Then there is $f \in \mathcal{A}$, such that $A_{n} f \nrightarrow f$ in the topology of $\mathcal{A}$.

In the next definition we introduce the notion of a $\delta$-interpolating collection of points, e.g. [D]. 
Definition 5. The collection $Z_{n}=\left\{z_{i}\right\}_{i=1}^{n}$ of points in the unit disk $\mathbf{D}$ is called $\delta$-interpolating iff

$$
\delta=\inf \left\{\prod_{i=1, i \neq k}^{n}\left|\frac{z_{k}-z_{i}}{1-\bar{z}_{i} z_{k}}\right| ; k=1,2, \ldots, n\right\} .
$$

The following theorem is due to Beurling and it shows how one can use the number $\delta$ from the above definition in order to estimate the norm of a certain interpolating operator.

Theorem (C). If $Z_{n}=\left\{z_{i}\right\}_{i=1}^{n}$ is $\delta_{n}$-interpolating, $n=1,2, \ldots$, then there exist functions $\left\{f_{j}\right\}_{j=1}^{n}, f_{j} \in \mathcal{A}$, such that

$$
\begin{gathered}
f_{j}\left(z_{i}\right)=\delta_{i j} ; \\
\sup \left\{\sum_{i=1}^{n}\left|f_{i}(z)\right| ; z \in \mathbf{D}\right\}=O\left(\frac{1}{\delta_{n}} \log \left(\frac{1}{\delta_{n}}\right)\right), \quad \text { as } n \rightarrow \infty .
\end{gathered}
$$

Remark. A simple corollary from this theorem is that the operator $W_{n}: l_{\infty}^{n} \rightarrow$ $\mathcal{A} ; W_{n}\left(\left(x_{i}\right)_{i=1}^{n}\right)=\sum_{i=1}^{n} x_{i} f_{i}(z)$ has its norm

$$
\left\|W_{n}\right\|=O\left(\frac{1}{\delta_{n}} \log \left(\frac{1}{\delta_{n}}\right)\right) \quad \text { as } n \rightarrow \infty .
$$

It is easy to show, using the same method as in [Sh], that if $Z_{n}=\left\{z_{k, n}\right\}_{k=1}^{n}$ is $\delta$-interpolating with the same constant $\delta>0$ for every $n=1,2, \ldots$, then Theorem 3 holds.

Definition 6. A collection of sets $Z_{n}=\left\{z_{k, n}\right\}_{k=1}^{m_{n}}, \quad n=1,2, \ldots$, in $\mathbf{D}$ is called uniformly Carleson iff the measures $\mu_{n}$ that assign a mass $1-\left|z_{k, n}\right|$ to every point $z_{k, n}, k=1,2, \ldots, n$, are uniformly Carleson, i.e. there is a constant $C$ such that $\frac{\mu_{n}\left(S_{h}(x)\right)}{h} \leq C$ for every $n \geq 1$, every $h \in(0,1)$, and every $x$-real. Here $S_{h}(x)=$ $\left\{r e^{i} \theta: 1-h<r<1,|x-\theta|<\frac{h}{2}\right\}$.

The next proposition, e.g. [Ni], gives another characterization of a uniform Carleson collection of points in the unit disk.

Proposition 2. The sets $Z_{n}=\left\{z_{k, n}\right\}_{k=1}^{m_{n}} \subset \mathbf{D}, n \geq 1$, are uniformly Carleson if and only if there exists constants $\delta>0$ and an integer $K>0$ such that

$$
\left\{Z_{n}\right\}=\bigcup_{i=1}^{K}\left\{Z_{i, n}\right\}
$$

where each $Z_{i, n}$ is $\delta$-interpolating. The constant $\delta$ and $K$ can be chosen to depend on the Carleson constant $C$ only.

\section{Proofs}

The idea of the proof of Theorem $\mathbf{3}$ is to assume that the recovery problem can be solved, and show that it implies $\gamma_{\infty}\left(L_{n}\right)=O(1)$, which together with Proposition 1 leads to a contradiction.

Proof of Theorem 3. Assume that there is a sequence of linear discrete operators $L_{n} \in R\left(\left\{Z_{n}\right\}\right)$ such that $L_{n} f \rightarrow f$ for every $f \in \mathcal{A}$. Since $\left\{Z_{n}\right\}$ is uniformly Carleson, we have by Proposition 2 that (7) holds. Moreover, we may assume 
without loss of generality that $\left\{Z_{n}\right\}=T_{n} \bigcup_{i=1}^{K}\left\{Z_{i, n}\right\}$ where $T_{n}=\left\{t_{i, n}\right\}_{i=1}^{k_{n}} \subset T$ (the unit circle) for otherwise we can consider $L_{n}$ as

$$
L_{n} f=\sum_{i=1}^{k_{n}} f\left(t_{i, n}\right) 0+\sum_{k=1}^{m_{n}} f\left(z_{k, n}\right) l_{k, n}, \quad f, l_{k, n} \in \mathcal{A} .
$$

Next consider the Blaschke product $B_{n}$ with zeros the points in $\bigcup_{i=1}^{K}\left\{Z_{i, n}\right\}$, and let $P_{n}$ be a projection on $\mathcal{A}$ generated by $B_{n}$, i.e. $\operatorname{ker} P_{n}=\left\{f \in \mathcal{A}: f=B_{n} g\right.$, for some $g \in \mathcal{A}\}$.

The selection of a proper $P_{n}$ is based on a simple proposition (cf. [CPS]) saying that if $P_{1}$ and $P_{2}$ are two projections on $\mathcal{A}$ with kernels generated by the Blaschke products $B_{1}$ and $B_{2}$ respectively, then one can find a projection $P$ on $\mathcal{A}$ with a kernel generated by $B_{1} B_{2}$, and the norm satisfying

$$
\|P\| \leq\left\|P_{1}\right\|+\left\|P_{2}\right\| \text {. }
$$

Applying this proposition $K$ times we obtain

$$
P_{n} f=P_{K, n} h_{K, n}+B_{K, n} P_{K-1, n} h_{K-1, n}+\ldots+B_{K, n} \ldots B_{2, n} P_{1, n} h_{1, n} .
$$

Here $P_{i, n}$ is a projection on $\mathcal{A}$ generated by the points in $Z_{i, n}, B_{i, n}$ are the Blaschke products with zeros the points in $Z_{i, n}$, and the functions $h_{i, n}$ are defined inductively as follows:

$$
h_{K, n}=f, \quad h_{K-i, n}=\frac{1}{B_{K-i+1, n}}\left(I-P_{K-i+1, n}\right)(f), \quad i=1, \ldots, K-1 .
$$

Now using (8), (9), Beurling's Theorem and the fact that $Z_{i, n}$ is $\delta$-interpolating for all $i=1, \ldots, K$, we obtain

$$
\left\|P_{n}\right\| \leq C_{1}
$$

where $C_{1}$ is a constant that depends on $K$ and $\delta$ only.

Next consider the operators $A_{n}: \mathcal{A} \rightarrow l_{\infty}^{m_{n}+k_{n}}$ defined as

$$
A_{n} f=\left(g_{n}\left(t_{1, n}\right), \ldots, g_{n}\left(t_{k_{n}, n}\right), h_{1, n}\left(z_{1,1, n}\right), \ldots, h_{K, n}\left(z_{K, 1, n}\right), \ldots, h_{K, n}\left(z_{K, l_{K}, n}\right)\right) .
$$

Here $g_{n, f}=\frac{1}{B_{n}}\left(I-P_{n}\right)(f), I$ is the identity operator, and $Z_{i, n}=\left\{z_{i, j, n}\right\}_{j=1}^{l_{i}}$ $\left(i=1, \ldots, K, l_{1}+\ldots+l_{K}=m_{n}\right)$.

Obviously $g_{n}, h_{i, n} \in \mathcal{A}, i=1, \ldots, K$, and applying the maximum modulus principle for analytic functions and Beurling's Theorem for $P_{i, n}, i=1, \ldots, K$, we get from (10) (recall that $\left|B_{n}(z)\right| \equiv 1,\left|B_{i, n}(z)\right| \equiv 1, \quad i=1, \ldots, K$, on $\mathbf{T}$ ) an estimate for the norms of the operators $A_{n}$

$$
\left\|A_{n}\right\| \leq C_{2} .
$$

Here $C_{2}$ is a constant that depends on $K$ and $\delta$ only.

Next introduce operators $V_{n}: l_{\infty}^{k_{n}+m_{n}} \rightarrow \mathcal{A}$ :

$$
\begin{aligned}
& V_{n}\left(v_{1}, \ldots, v_{k_{n}}, w_{1,1}, \ldots, w_{1, l_{1}}, \ldots, w_{K, 1}, \ldots, w_{K, l_{K}}\right) \\
& \quad=B_{n}(z) \sum_{i=1}^{k_{n}} v_{i} g_{i, n}(z)+\sum_{j=1}^{K}\left\{\frac{B_{n}}{B_{1, n} \ldots B_{j, n}}(z) \sum_{i=1}^{l_{j}} w_{j, i} f_{j, i, n}(z)\right\} .
\end{aligned}
$$

Here $f_{j, i, n}(z) \in \mathcal{A}$ are such that $P_{j, n} f(z)=\sum_{i=1}^{l_{j}} f\left(z_{j, i, n}\right) f_{j, i, n}(z), f_{j, i, n}\left(z_{j, l, n}\right)=$ $\delta_{i l}, \sup \left\{\sum_{i=1}^{l_{j}}\left|f_{j, i, n}(z)\right|, z \in \mathbf{D}\right\}=\left\|P_{j, n}\right\|$, and $w_{i, n}$ 's are selected in such a way (e.g. ISh] proof of Theorem 2) that $\sum_{i}\left\|g_{i, n}\right\| \leq M$, where $M$ is an absolute constant. 
From this applying the maximum modulus principle for analytic functions and the fact that the absolute value of a Blaschke product equals 1 on $\mathbf{T}$ we obtain

$$
\left\|V_{n}\right\| \leq C_{3} \text {. }
$$

Here the constant $C_{3}$ depends on $K, M$ and $\delta$ only. Set $Q_{n}=V_{n} A_{n}$. By the construction of the projection $P_{n}$ we have

Obviously

$$
\begin{gathered}
Q_{n} f\left(z_{1, i, n}\right)=P_{n} f\left(z_{1, i, n}\right)=f\left(z_{1, i, n}\right), \quad i=1, \ldots, l_{1}, \\
\ldots \\
Q_{n} f\left(z_{K, i, n}\right)=P_{n} f\left(z_{K, i, n}\right)=f\left(z_{K, i, n}\right), \quad i=1, \ldots, l_{K} .
\end{gathered}
$$

$$
Q_{n} f\left(t_{i, n}\right)=f\left(t_{i, n}\right), \quad i=1, \ldots, k_{n},
$$

holds as well. Thus $L_{n} Q_{n} f=L_{n} f, f \in \mathcal{A}$ and $\gamma_{\infty}\left(L_{n}\right) \leq\left\|L_{n}\right\|\left\|V_{n}\right\|\left\|A_{n}\right\|$. Since $L_{n} f \rightarrow f$, as $n \rightarrow \infty$ for every $f \in \mathcal{A},\left\|L_{n}\right\|$ are uniformly bounded, and combining this with the observation that (11) and (12) imply $\gamma_{\infty}\left(Q_{n}\right)=O(1)$. we obtain from the above estimate

$$
\gamma_{\infty}\left(L_{n}\right)=O(1)
$$

This last estimate allows us to apply Proposition 1 to contradict $L_{n} f \rightarrow f$.

For the proof of Theorem 4 we need the following:

Proposition 3. Let $\left\{B_{n}\right\}$ be a sequence of finite Blaschke products with zero sets equal to $\left\{Z_{n}\right\}, n \geq 1$, respectively, and assume that the collection $\left\{Z_{n}\right\}, n \geq 1$, is not uniformly Carleson. Furthermore let $\epsilon>0$ and the positive integer $N$ are given.

Then there is an integer $m$ and a point $z_{0} \in \mathbf{D}^{\mathbf{o}}$ such that

$$
B_{m}=B_{1, m} \ldots B_{N, m} \quad \text { and } \quad\left|B_{j, m}\left(z_{0}\right)\right|<\epsilon \quad \text { for } j=1, \ldots, N,
$$

where the $B_{j, m}$ 's are Blaschke products.

Proof. The following proof is an adaptation of an argument one can find in CPS. Since $\left\{Z_{n}\right\}, n \geq 1$, is not uniformly Carleson, we can find for every $N$ and for every constant $C$ infinitely many positive integers $m=m(C)>N$, numbers $h=h(C) \in$ $(0,1)$ and $x=x(C)$-real, such that

$$
\sum_{z_{k, m} \in S_{h}(x)}\left(1-\left|z_{k, m}\right|\right)>C h .
$$

Here we may assume without loss of generality that $0<h<\frac{1}{3}$. Notice that for $t \in[1,2]$

$$
\log t \geq \frac{\log 2}{3}\left(t^{2}-1\right)
$$

and thus for $z \in S_{h}(x)$ and for $z_{0}=(1-3 h) e^{i x}$ we have

$$
\log \left|\frac{1-\bar{z} z_{0}}{z-z_{0}}\right| \geq \frac{1-|z|^{2}}{h} \frac{\log 2}{16} .
$$

Now, if $A_{m}$ is the Blaschke product generated by those zeros $w_{k}$ of $B_{m}$ that lie in $S_{h}(x)$, we get

$$
\left|A_{m}\left(z_{0}\right)\right| \leq \exp \left(-\frac{\log 2}{8} C\right)
$$


Since $h$ is assumed to satisfy $0<h<\frac{1}{3}$ we have for every $z \in S_{h}(x)$

$$
\left|\frac{z-z_{0}}{1-\bar{z} z_{0}}\right| \geq \frac{1}{2}
$$

If we pick $C>0$ such that $\exp \left(-\frac{\log 2}{8} C\right)<\left(\frac{\epsilon}{2}\right)^{N}$, then for $m=m(C)$ we get from (15) that

$$
\left|A_{m}\left(z_{0}\right)\right| \leq\left(\frac{\epsilon}{2}\right)^{N}
$$

This together with (16) implies that we can find an $l_{1}$ such that

$$
\frac{\epsilon}{2}<\prod_{k=1}^{l_{1}}\left|\frac{w_{k}-z_{0}}{1-\bar{w}_{k} z_{0}}\right|<\epsilon .
$$

Set

$$
B_{1, m}(z)=\prod_{k=1}^{l_{1}} \frac{w_{k}-z}{1-\overline{w_{k}} z} .
$$

Now it is easy to see that

$$
\left|\frac{A_{m}\left(z_{0}\right)}{B_{1, m}\left(z_{0}\right)}\right|<\left(\frac{\epsilon}{2}\right)^{N-1} .
$$

Therefore there is an $l_{2}$ such that

$$
\frac{\epsilon}{2} \leq \prod_{k=l_{1}+1}^{l_{2}}\left|\frac{w_{k}-z_{0}}{1-\bar{w}_{k} z_{0}}\right|<\epsilon
$$

and we can set

$$
B_{2, m}(z)=\prod_{k=l_{1}+1}^{l_{2}} \frac{w_{k}-z}{1-\overline{w_{k}} z} .
$$

Obviously we can continue in the same fashion (recall that $m>N$ ), obtaining Blaschke products $B_{3, m}, B_{4, m}, \ldots, B_{N-1, m}$, and for each one of them $\left|B_{j, m}\left(z_{0}\right)\right|<\epsilon$. Moreover

$$
\left|\frac{A_{m}\left(z_{0}\right)}{B_{1, m}\left(z_{0}\right) \ldots B_{N-1, m}\left(z_{0}\right)}\right|<\epsilon .
$$

Now recall that $A_{m}$ divides $B_{m}$, and set

Obviously

$$
B_{N, m}=\frac{B_{m}}{B_{1, m} \ldots B_{N-1, m}} \text {. }
$$

and the proposition is proved.

$$
\left|B_{N, m}\left(z_{0}\right)\right|<\epsilon
$$

Proof of Theorem 4. Consider two cases:

First if $\left\{Z_{n}\right\}$ happens to be a uniform Carleson collection of points, then Theorem 3 implies Theorem 4.

Second if $\left\{Z_{n}\right\}$ is not a uniformly Carleson collection of points, assume to the contrary that there are projections $P_{n} \in R\left(Z_{n}\right)$ such that for every $f \in \mathcal{A}, P_{n} f \rightarrow f$, as $n \rightarrow \infty$. This in turn implies that $\left\|P_{n}\right\|=O(1)$, and thus one can find an $\epsilon>0$ such that for every $n \geq 1$

$$
\epsilon\left\|I-P_{n}\right\|<\frac{1}{2}
$$


By Proposition 3, if $\delta>0$ and $N>0$ are given, we can find $m>N$ and $z_{0} \in \mathbf{D}^{\mathbf{o}}$ so that $B_{m}=B_{1, m} \ldots B_{N, m}$, and every $B_{j, m}$ satisfies $\left|B_{j, m}\left(z_{0}\right)\right|<\delta$. Set

$$
F_{m}(z)=\prod_{k=1}^{N} \frac{B_{k, m}(z)-B_{k, m}\left(z_{0}\right)}{1-\bar{B}_{k, m}\left(z_{0}\right) B_{k, m}(z)} .
$$

Obviously $F_{m}$ is an inner function having zero of multiplicity at least $N$ at $z_{0}$. If we take $\delta$ small enough we see that $\left\|F_{m}-B_{m}\right\|_{\infty}<\epsilon$. An easy proposition (cf. CPS] Proposition 7) shows that in this case we can find a projection $Q_{m}$ on $\mathcal{A}$ with the norm

$$
\left\|Q_{m}\right\| \leq 2 \sup \left\{\left\|I-P_{m}\right\|\right\}
$$

such that $z_{0}$ (with multiplicity at least $N$ ) is one of the points that generate $Q_{m}$, i.e. $k e r Q_{m}=\left\{f \in \mathcal{A}: f=F_{m} g, g \in \mathcal{A}\right\}$. Therefore one can find an absolute constant $J$ (cf. [CPS] Proposition 6) such that

$$
\left\|Q_{m}\right\| \geq J \log N \text {. }
$$

Since $N$ is arbitrary (19) contradicts (17) and (18) and the poof of Theorem 2 is complete.

As an immediate application of Theorem 2 we obtain

Corollary 1. $\mathcal{A}$ does not possess an interpolating basis.

\section{REFERENCES}

[Bo] S. Bochkariev (1985): Construction of interpolating basis in the space of continuous functions based on the Fejer kernel, MIAN, SSR 172, 29-59. MR 87a:41004

[C] L. Carleson (1958): Research on interpolation problems, Air Force Technical Report, AF 61(052)-238, AD 278-144.

[CPS] P. Casazza, R. Pengra and C. Sundberg (1980): Complemented ideals in the disk Algebra, Israel J. Math., 37: 76-83. MR 82d:30029

[D] P. Duren (1970): Theory of $H^{p}$ spaces, Academic Press. MR 42:3552

[ISh] I. Ivanov and B. Shekhtman (1998): Linear Discrete Operators and Recovery of Functions, Proceedings of Approximation Theory IX, Vol 1: 159-166. CMP 2000:11

[LR] J. Lindenstrauss and H. Rosenthal (1969): The $\mathcal{L}_{p}$ spaces, Israel J. Math., 7:325-349. MR 42:5012

[Ni] N. Nikol'skii (1986): Treatise on the Shift Operator, Springer- Verlag, Berlin Heidelberg. MR 87i:47042

[P] J. Partington (1997): Interpolation, Identification and Sampling, Clarendon Press, Oxford. MR 99m:41001

[Sh] B. Shekhtman (1992): Discrete approximating operators on function algebras, Constructive Approximation 8: 371-377. MR 93h:46071

[So] G. Somorjai (1980): On discrete operators in the function space $\mathcal{A}$, Constructive Approximation Theory 77, Sofia, 489-496.

[To] V. Totik (1984): Recovery of $H^{p}$ - Functions, Proceedings of the American Mathematical Society, Vol. 90, 531-537. MR 85j:30073

Department of Mathematics, Texas A \& M University, College Station, Texas 77843

E-mail address: ivanov@math.tamu.edu

Department of Mathematics, University of South Florida, Tampa, Florida 33620

E-mail address: boris@2chuma.cas.usf.edu 